\title{
Middle School Physics Teachers' Content Knowledge of Acceleration
}

\author{
Elijah Tabachnick, ${ }^{1}$ Peter Colesworthy, ${ }^{2}$ Michael C. Wittmann ${ }^{1,2}$ \\ ${ }^{1}$ University of Maine, Department of Physics and Astronomy, 5709 Bennett Hall, Orono, ME, 04469-5709 \\ ${ }^{2}$ University of Maine, Center for Research in STEM Education, 5727 Estabrooke Hall, Orono, ME, 04469-5727
}

\begin{abstract}
In the "speed model" of accelerated motion, the terms "speeding up" and "slowing down" are equated with positive and negative acceleration, respectively. As part of the Maine Physical Sciences Partnership, we have investigated middle school physical science teachers' understanding of accelerated motion in the context of using vectors as a pictorial tool for kinematics and found a high prevalence of the speed model. Through surveys, interviews, and observation of professional development activities, we have found that the teachers consistently use the correct mathematical tools to talk about displacements and velocities, and correctly use vectors to represent displacements, velocities and accelerations. However, when interpreting the acceleration of an object, teachers often use the speed model, which contradicts their other work. We discuss this result and present two conjectures about its possible origin.
\end{abstract}

\section{INTRODUCTION}

This study focuses on middle school physical science teachers' content knowledge of accelerated motion. Researchers in physics education have studied kinematics extensively, and teaching acceleration has been shown to be difficult at many levels of instruction [1-4]. In the context of the Maine Physical Sciences Partnership (MainePSP), we have continued previous work to study teacher knowledge of force and motion [5], in particular accelerated motion [6]. Previous studies of teachers in the Maine STEM partnership revealed the use of a model that describes acceleration in the direction of velocity as positive, and acceleration opposed to velocity as negative. More specifically, many students and teachers equate "speeding up" with positive acceleration and "slowing down" with negative acceleration. This is only true if velocity is positive, which is often the case in situations where an object is moving to the right and the associated coordinate system is positive to the right, as well. We call this model the "speed model," while we call the formal physics analysis based on vectors within a coordinate system the "direction model."

In this paper we describe data collected from four teachers, three of whom were surveyed, interviewed, and observed in professional development (PD) activities, with the fourth observed only during PD activities. The goal of data collection was, first, to identify teachers' knowledge pieces in other kinematics contexts (such as velocity problems), and, second, to see how those teachers solved acceleration problems and if they used the same knowledge pieces in solving the acceleration problems.

\section{MOTIVATION}

Acceleration is a vector quantity and as such requires understanding of vectors, as well as coordinate systems and the mathematical operations of addition and subtraction of vectors. According to the K-12 Framework [7] that underlies the Next Generation Science Standards [8], at the eighth grade level, "all positions of objects and the directions of forces and motions must be described in an arbitrarily chosen reference frame" (p.115) [7]. Whereas students at the end of $5^{\text {th }}$ grade are explicitly required not to use vectors in reasoning about the relationship between force and motion, $8^{\text {th }}$ grade standards have no such restriction (p.115) [7]. Regarding the vector operations around accelerated motion, and their translation into a coordinate system, we expect students solving problems in only one dimension to be able to work with positive and negative numbers consistent with the Common Core Standards [9]. In sum, students should be able to analyze a problem with an arbitrary combination of a one dimensional coordinate system pointing either left or right, an object moving to the left or right, and speeding up or slowing down.

We expect that teachers understand the material they teach at least as well as the students they are teaching. We investigated teachers' content understanding of kinematics in one dimension. To do so, we asked them questions about displacement, velocity, and acceleration. We looked at ideas common to all three areas, such as vector addition and subtraction. We analyze their results in terms of a resources framework $[10,11]$. The resources framework breaks ideas into pieces which independently are neither right nor wrong. In this way we can highlight what resources teachers have for solving acceleration problems and see if they apply them appropriately to the problems we ask. This framework helps us formalize the context-dependence we observed [12]. We found that teachers could correctly use resources related to vectors and signs within a coordinate system in the context of questions about displacements and velocities, but when asked about acceleration they did not use these resources and reverted back to the "speed model" to determine the sign of acceleration. 


\section{DATA COLLECTION}

Data were collected from surveys, interviews and PD sessions. The survey was distributed on paper to allow for drawing of vectors and open-ended written responses. The first section of the survey was about the terms direction and magnitude as they relate to vectors and allowed the researchers to see if the teachers were familiar with basic vector concepts. The second section was a group of questions surrounding a single physical situation where a ball with an initial velocity approaches a fan, turns around and then speeds up away from the fan (Fig. 1).

Interviews were made up of questions about uniform and non-uniform motion. First, after teachers were given a chance to practice thinking aloud [13] as they explained their answers, teachers were shown two stop-motion diagrams representing uniform motion in opposite directions, and they were asked to describe what was happening. The goal was to get the teachers thinking about vectors to describe motion, identifying uniform motion with vectors, and identifying a difference in direction as a difference in velocity. Non-uniform motion was represented by the mirror image of the survey question. Teachers were asked to draw and describe displacement, velocity, and acceleration vectors with the ball now coming in from the right, reversing direction, and going out to the left.

In the PD session, teachers discussed an experimental set-up with a coordinate system assigned by the facilitator of the session (author MCW). A ball with negative acceleration was speeding up going down a very long and shallowly tilted incline (Fig. 2). This scenario allowed us to see how teachers used positive and negative when talking about velocity vectors, and whether they used the same reasoning resources when talking about acceleration vectors.

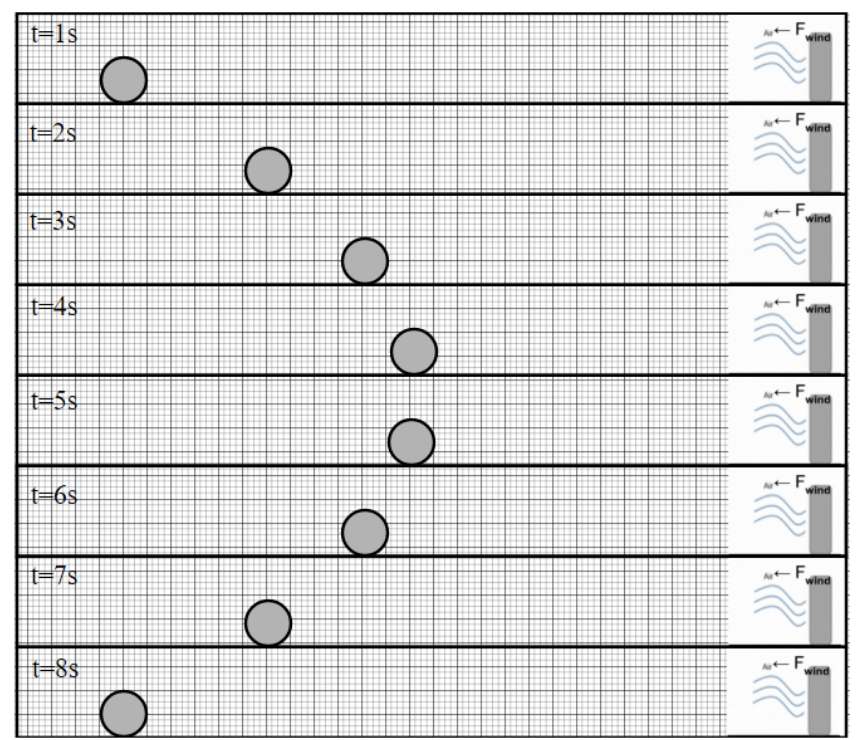

FIG 1. Survey question with ball reversing directions due to a constant force. The interview question was left-right mirrored, with the ball coming in from the right.

\section{RESULTS}

In previous work, Kranich et al. found that some teachers used the speed model to solve acceleration problems [6]. The question was whether they did not have the tools to analyze acceleration correctly, or if other issues were causing them problems. In our study, we observed the use of resources which are productive and necessary to solve kinematics problems. However, the teachers reverted to using the speed model when answering acceleration problems. We summarize the results of the survey and interview for three teachers (Alice, Bob, and Carl, all aliases) and then discuss the individual work of the three teachers across the survey and interview. At the end of this section, we discuss the PD session that involved a fourth teacher (Dani).

The survey showed that the teachers understood the vector concepts of magnitude and direction in the context of displacement and velocity. From interviews, we also observed that all teachers could compare magnitudes of vectors when talking about displacement and velocity. Differences arose when we started to look at acceleration problems.

Alice, for example, correctly identified magnitude and direction of vectors on the survey, and constructed displacement vectors with proper labels for the given coordinate system on the second part of the survey. In the interview, she constructed displacement vectors and used an east-west coordinate system to label direction. She abstracted the displacements to average velocity vectors. In both situations, however, Alice did not apply the construction of vectors to a description of the acceleration. After finding the change in the velocity vectors on the survey, Alice answered the question about positive and negative acceleration by saying (as a reminder, the diagram in Fig. 1 is reversed in the interview)

"From $t_{1}$ through $t_{4}$ the acceleration is negative since it's in the opposite direction of the motion... and from $t_{5}$ through $t_{8}$ there is positive acceleration in the same direction as motion."

This change in the sign of the acceleration is consistent with the speed model, negative when slowing down and positive when speeding up. The same results appeared in the interview when Alice had drawn velocities and used her east-west coordinate system to label them, and found the change in velocity to be the same magnitude and in the

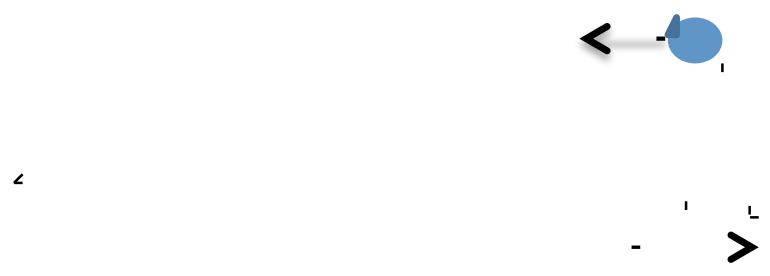

FIG 2. Ball rolling to the left down a ramp, with a coordinate system defined as positive to the right. 
same direction throughout the motion, "Well, here the ball is slowing down so that is negative acceleration. Right?" Alice used her own coordinate system (in terms of east and west motion) when discussing velocity, but used positivenegative labels for acceleration, consistent with the speed model for solving acceleration problems.

Bob also used the speed model consistently, but could use vector operations to find average velocity. On the survey, he used vectors to talk about displacement and velocity within the defined coordinate system of the survey. In the interview, he used a positive-negative coordinate system. In the interview, while using a positive-negative coordinate system, he stated, "so in the first half of the motion the ball is slowing down, so that's negative acceleration, and after the turn around it's speeding up so that's positive acceleration." His tone of voice suggested that he was confident in the speed model and he spoke of this model as how these problems were always solved.

Carl gave conflicting answers on his survey. He said that the first half of the motion was slowing down, so the ball had negative acceleration, and the second half was speeding up so the ball had positive acceleration. However, when asked if a single vector could describe the acceleration, he said yes, because the acceleration was the same magnitude and direction for the entire motion. Carl did not make note of a conflict between his two statements. In the interview, we found that Carl used the direction model. We believe that Carl responded to the structured questioning of the interview in such a way that he used more appropriate reasoning about motion within a coordinate system, but we are unable to say why he responded as he did while the other interviewed teachers did not.

Our information about Dani comes from the PD session in which teachers discussed the experimental set-up in Fig. 2. The PD session involved pre-service and in-service teachers. We will describe the general set of events involving the whole group before describing her responses, specifically. As before, we observed teachers drawing and properly labeling displacement vectors and talking about them with the correct direction language, using these vectors to discuss average velocities over equal time intervals. Then teachers made change in velocity vectors, pointing in the negative direction. However, when asked about the sign of acceleration, there was a certainty among the teachers we were studying that the acceleration had to be positive, because the ball was speeding up. This is incorrect, based on the motion within the coordinate system shown in Fig. 2 and their own change in velocity vectors, which over equal time intervals they knew had to represent the acceleration. Teachers had drawn the change in velocity vectors and talked about how all were pointing in the same direction (negative), and over equal time intervals, however, they did not use the same vector resources used to answer the question about acceleration. Instead, they gave an answer consistent with the speed model.
Teachers were then reminded that the velocity vectors they had been looking at previously pointed in this same direction yet we called those vectors negative. Dani responded by saying

"It doesn't matter because we're not talking about displacement; we're talking about change in velocity. So the speed is getting larger, then the acceleration is positive."

Because the PD session was coming to an end, and the PD leader (author MCW) did not want to have teachers leave without discussing the formal answer, he described the answer, cutting off time for teachers themselves to arrive at the conclusion (namely, the ball was speeding up, but with negative acceleration).

Afterward, Bob was the first to speak, saying, "If we had it the other way around, and were slowing down, would that be positive acceleration?" followed by an uncomfortable laugh of disbelief. He had made a correct point without seeming to believe it; he was fully capable of using the direction model yet did not believe that it was correct. A brief conversation followed with Dani and Bob in which his question was affirmed (yes, that is positive acceleration, but it's slowing down). He expressed disbelief that this could be the case, but that it was consistent with the work they had done during the PD session. In a later conversation, Dani reported thinking about this problem on her hour-long drive home from the PD session, and continuing to think about it and discuss it with colleagues for days.

\section{DISCUSSION}

Knowledge for teaching is a complex topic. We expect teachers to know content, how their students are thinking, how to think about content in a way that best matches student needs, and how to create an effective classroom in which all can learn $[14,15]$. In terms of content knowledge, we expect our teachers to know the physics that is appropriate for teaching the ideas at the appropriate grade level. Many middle school teachers are teaching out of field and do not have a strong physics background. As Kranich et al. discussed [6], when using only coordinate systems with positive to the right, and objects moving to the right, this does not lead to problems. But, such a limited choice of motion and coordinate systems is inconsistent with the goals of the NGSS.

We found that teachers were able to use productive resources for understanding acceleration, mostly in the context of finding average velocities. All four teachers had some familiarity with vectors and used them appropriately in the context of displacement and velocity questions. But, they did not use these resources when solving acceleration problems. Instead, they all used the speed model at least sometimes. One teacher, Carl, realized that the vectors he had drawn implied there was only one acceleration for the motion of the ball in Fig. 1, but when using signs and the 
language of speeding up and slowing down, he still used the speed model.

It seems that teachers have a definition of positive and negative acceleration that is inconsistent with physics formalism. One conjecture to help us think about how teachers think about this model is that in everyday experience, we almost always experience motion as positive while walking forward. People walking forwards likely consider their own displacement to always be positive, and therefore the speed model would always be correct. We think of this is a person-centric coordinate system (with velocity always positive and forward when walking normally). No matter which direction one moves, speeding up is positive acceleration, and slowing down (without turning around) is negative acceleration.

A second conjecture for how teachers use the speed model, even when it contradicts how they arrived at their responses for average velocity, is an issue related to mathematics. In vector terms, $\mathbf{v}_{\mathbf{f}}=\mathbf{v}_{\mathbf{i}}+\mathbf{a t}$ (bold for vector quantities). The sign is always positive, even when $\mathbf{v}_{\mathbf{i}}$ and $\mathbf{a}$ are in opposite directions. In scalar terms, though, when the value of the velocity decreases, it is as if something was subtracted from the initial velocity. This subtraction, a minus sign, might get ascribed to the acceleration (as a negative sign), meaning that slowing down is identical to negative acceleration. We have suggestions of this explanation from interviews with a teacher not described in this paper, because we only had limited data about her reasoning. Our conjecture is consistent with past work by Hayes and Wittmann [16].

Further research is needed to explore these two conjectures, as well as others.

[1] R. D. Knight, Phys. Teach., 33, 74 (1995).

[2] P. S. Shaffer and L. C. McDermott, Am. J. Phys., 73, 921 (2005).

[3] F. Reif and S. Allen, Cog. Instr., 9, 1 (1992).

[4] D. E. Trowbridge and L. C. McDermott, Am. J. Phys., 49, 242 (1981).

[5] D. P. Laverty, Master of Science in Teaching thesis, U. Maine, 2015. http://digitalcommons.library. umaine.edu/etd/2410. Retrieved 10/2/2017.

[6] G. D. Kranich, M. C. Wittmann, and C. Alvarado, 2015 PERC Proceedings [College Park, MD, July 2930, 2015], edited by A. D. Churukian, D. L. Jones, and L. Ding, doi:10.1119/perc.2015.pr.040.

[7] NGSS Lead States, Next Generation Science Standards: For States, by States (The National Academies Press, Washington, DC, 2013).

[8] National Research Council, A Framework for K-12 Science Education: Practices, Crosscutting Concepts, and Core Ideas (The National Academies Press, 2012).

\section{CONCLUSIONS}

We were able to observe teachers in a variety of contexts answering questions about displacement, velocity, and acceleration. We observed that they had the tools to answer acceleration questions using vector differences, based on their ability to answer velocity questions, but that they did not do so. Instead, many teachers used the speed model, at least some of the time. When confronted with the direction model, some teachers expressed surprise and some level of consternation. Others used both the speed and the direction model but did not notice the contradiction involved.

We suggest that teachers need more experience with developing these ideas. The NGSS sets a difficult goal for middle school teaching, essentially a full vector-based model of Newton's Second Law. Teachers need to have the skill to teach these ideas. Further work is needed to identify productive teacher resources and help them recognize their utility, thereby improving their own knowledge of the content that they teach their students.

\section{ACKNOWLEDGEMENTS}

We thank Greg Kranich and Laura Millay for their help with the research design and implementation. We also thank the teachers of the Maine Physical Sciences Partnership who participated in the activity. This work was supported in part by NSF grant MSP-0962805.
[9] National Governors Association Center for Best Practices, Council of Chief State School Officers, Common Core State Standards (Mathematics) (National Governors Association Center for Best Practices, Council of Chief State School Officers, 2010).

[10] D. Hammer, Am. J. Phys., 68, S45 (2000).

[11] M. C. Wittmann, Phys. Rev. ST - PER, 2, 20105 (2006).

[12] R. E. Scherr, Am. J. Phys., 75, 272 (2007).

[13] W. M. Someren, F. Y. Barnard, and A. C. J. Sandberg, The Think Aloud Method. A Practical Guide to Modeling Cognitive Process (Harcourt Brace \& Company, 1994).

[14] D. L. Ball, M. H. Thames, and G. Phelps, J. Teach. Educ., 59, 389 (2008).

[15] S. Magnusson, J. Krajcik, and H. Borko, in Examining Pedagogical Content Knowledge: The Construct and Its Implications for Science Education, edited by J. Gess-Newsome and N. G. Lederman, pp. 95-132 (Kluwer Academic Press, 1999)

[16] K. Hayes and M. C. Wittmann, Phys. Teach., 48, 246 (2010). 